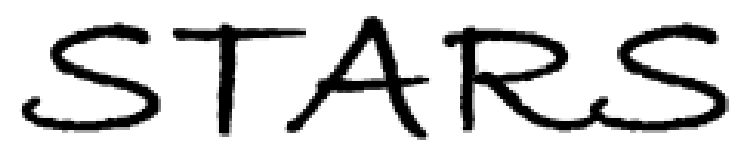

University of Central Florida

STARS

$1-1-2008$

\title{
A model for self-defocusing in laser drilling of polymeric materials
}

Chong Zhang

University of Central Florida

Nathaniel R. Quick

Aravinda Kar

University of Central Florida

Find similar works at: https://stars.library.ucf.edu/facultybib2000

University of Central Florida Libraries http://library.ucf.edu

This Article is brought to you for free and open access by the Faculty Bibliography at STARS. It has been accepted for inclusion in Faculty Bibliography 2000s by an authorized administrator of STARS. For more information, please contactSTARS@ucf.edu.

\section{Recommended Citation}

Zhang, Chong; Quick, Nathaniel R.; and Kar, Aravinda, "A model for self-defocusing in laser drilling of polymeric materials" (2008). Faculty Bibliography 2000s. 1200.

https://stars.library.ucf.edu/facultybib2000/1200

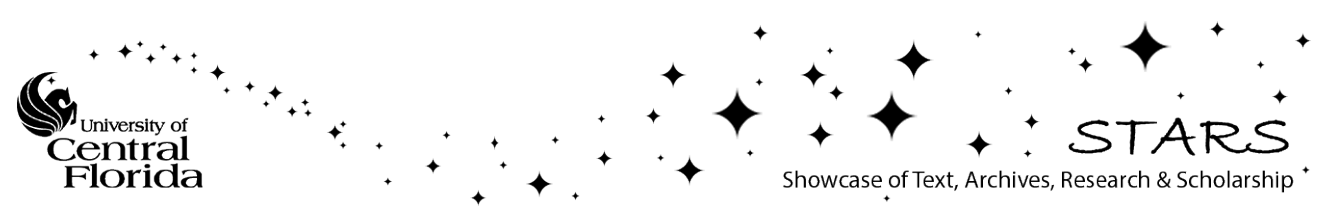




\section{A model for self-defocusing in laser drilling of polymeric materials}

Cite as: J. Appl. Phys. 103, 014909 (2008); https://doi.org/10.1063/1.2829818

Submitted: 04 June 2007 . Accepted: 08 November 2007. Published Online: 14 January 2008

Chong Zhang, Nathaniel R. Quick, and Aravinda Kar

\section{ARTICLES YOU MAY BE INTERESTED IN}

One-dimensional transient analysis of volumetric heating for laser drilling

Journal of Applied Physics 99, 113530 (2006); https://doi.org/10.1063/1.2204828

A complete model of keyhole and melt pool dynamics to analyze instabilities and collapse during laser welding

Journal of Laser Applications 26, 042001 (2014); https://doi.org/10.2351/1.4886835

Two-dimensional model for material damage due to melting and vaporization during laser irradiation

Journal of Applied Physics 68, 3884 (1990); https://doi.org/10.1063/1.346275

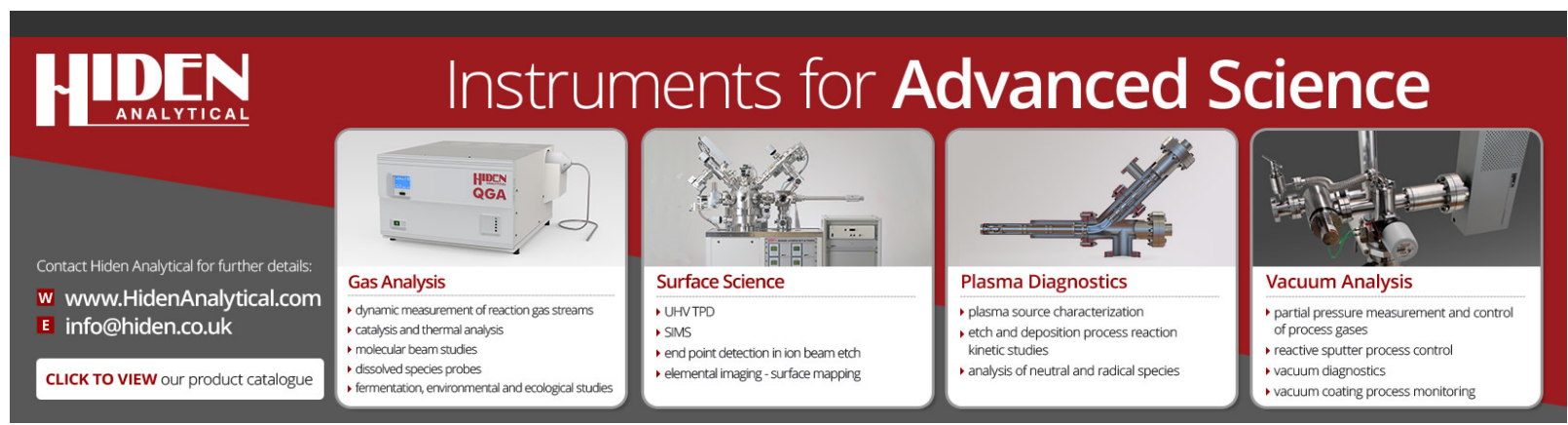




\title{
A model for self-defocusing in laser drilling of polymeric materials
}

\author{
Chong Zhang \\ Department of Mechanical, Materials and Aerospace Engineering, College of Optics and Photonics, \\ Center for Research and Education in Optics and Lasers (CREOL), University of Central Florida, \\ Orlando, Florida 32816-2700, USA \\ Nathaniel R. Quick \\ AppliCote Associates, LLC, 1445 Dolgner Place, Suite 23, Sanford, Florida 32771, USA

\begin{abstract}
Aravinda Kar ${ }^{\mathrm{a}}$
College of Optics and Photonics, Center for Research and Education in Optics and Lasers (CREOL), University of Central Florida, Orlando, Florida 32816-2700, USA
\end{abstract}

(Received 4 June 2007; accepted 8 November 2007; published online 14 January 2008)

A numerical thermal model is presented for laser microvias drilling in multilayer electronic substrates with Nd:YAG (YAG denotes yttrium aluminum garnet) and $\mathrm{CO}_{2}$ lasers. Such substrates have different optical properties such as the refractive index and absorption coefficient at these two laser wavelengths, resulting in different drilling mechanisms. Since the skin depth of the polymer is large for both the lasers, volumetric heating is considered in the model. As soon as a small cavity is formed during the drilling process, the concave curvature of the drilling front acts as a concave lens that diverges the incident laser beam. This self-defocusing effect can greatly reduce the drilling speed as predicted by the model. This effect makes the refractive index of the substrate at different wavelengths an important parameter for laser drilling. The model was used to calculate the laser ablation thresholds which were found to be 8 and $56 \mathrm{~J} / \mathrm{cm}^{2}$ for the $\mathrm{CO}_{2}$ and Nd:YAG lasers respectively. Due to the expulsion of materials because of high internal pressures in the case of Nd:YAG laser microvia drilling, the ablation threshold may be far below the calculated value. A particular laser beam shape, such as pitch fork, was found to drill better holes than the Gaussian beam. () 2008 American Institute of Physics. [DOI: 10.1063/1.2829818]

\section{INTRODUCTION}

Lasers are often used for high precision processing of semitransparent materials, such as polymer and semiconductor, for which the skin depth is large at certain laser wavelengths ${ }^{1}$ allowing deep penetration of the laser beam into the material. $\mathrm{CO}_{2}$ (10.6 or $9.3 \mu \mathrm{m}$ wavelength) and Nd:YAG (YAG denotes yttrium aluminum garnet) (wavelength $1.06 \mu \mathrm{m}$ ) lasers are prevalent in various industries. The photon energies of these lasers are much lower than the bond energies of most polymers and semiconductor materials. So photothermal ablation is the dominant mechanism for material removal, which is based on thermal processes such as melting and vaporization. The laser energy is absorbed inside the material, and therefore, volumetric heating occurs during laser drilling process. ${ }^{2}$ The volumetric heating may introduce thermomechanical breakage of the material due to large thermal stress which can degrade the hole quality. The temperature distribution due to volumetric heating needs to be analyzed to control the drilling process.

It is difficult to measure the temperature inside a material directly. Holographic interferometry can be used to measure the change in refractive index ${ }^{3}$ or thermal displacement ${ }^{4}$ to infer the temperature field. These techniques, however, are not widely used due to the complexity of the experimental setup and applicability to limited materials. Modeling is preferred to study the evolution of temperature fields inside the

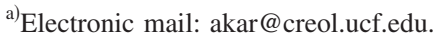

material during laser processing. Most of the analytical models are based on the surface absorption of the laser energy. ${ }^{5-9}$ Multiple reflections ${ }^{5,10}$ and geometrical effects on surface reflectivity ${ }^{11}$ are important for surface absorption in many materials such as metals. For large volumetric absorption of laser energy in transparent materials such as polymers, the surface reflectivity is small and multiple reflections can be neglected.

Usually numerical models are used to describe the volumetric heating process. Noguchi et al. ${ }^{12}$ applied the enthalpy method to formulate a one-dimensional volumetric heating model and solved it using a finite element technique. Voisey and Clyne ${ }^{13}$ and Sezer et al. ${ }^{14}$ numerically simulated pulsed laser drilling of thermal barrier coatings with assist gas. Semak et al. ${ }^{15}$ used a finite difference method, whereas Zeng et $a l .{ }^{16}$ presented an analytic model to calculate the temperature field during laser drilling by considering convective heat transfer due to the liquid metal flow induced by the recoil pressure of the outgoing metal vapor. The convective heat transfer is negligible in the case of polymeric materials for which the liquid layer is thin. ${ }^{2}$

Self-defocusing of the laser beam is another effect that can be important in laser drilling of semitransparent materials. As the drilling process progresses, a crater-shaped hole with a concave curvature is generally formed. The concave surface acts as a lens with negative focal length defocusing the incident laser beam. This phenomenon is termed as selfdefocusing during laser drilling, which depends on the re- 
fractive index of the material. Large refractive index means high optical power of the negative lens leading to more pronounced defocusing effect. Most of the studies analyzed the role of plasma on self-focusing or self-defocusing effects during laser-material interactions. ${ }^{17-19}$ Strombeck and $\mathrm{Kar}^{20}$ studied the self-focusing effect in laser welding where convex surfaces are formed by the molten material due to the surface tension between the melt and substrate.

This paper examines the self-defocusing effect arising due to the divergence of the incident laser beam by the concave drilling front as the laser beam propagates into the polymeric substrate. Volumetric laser heating is also considered in the thermal model which is solved using the finite difference method. Since the laser irradiance is very high for laser drilling, the volumetric heat source term in the energy equation generally causes numerical instability while solving the finite difference equations. To ensure computational stability, time-split MacCormack method ${ }^{21}$ is used. The locations of the drilling front are tracked at each time step of the calculation.

During laser drilling of polymers, the material removal may occur at a fixed thermal decomposition temperature due to several physicochemical phenomena such as the phase transitions (e.g., solid $\rightarrow$ glassy phase $\rightarrow$ melt $\rightarrow$ vapor), gas diffusion, chemical degradation, and chemical reaction. To account for the energy involved in this complex process, "specific ablation heat," representing the amount of heat needed to ablate a unit mass of the polymeric material at its thermal decomposition temperature, is included in the model in this study. This specific ablation heat is similar to the term "latent heat of vaporization" used to define the heat input for boiling materials at their respective boiling temperature. The effect of plasma is neglected in this study because the plasma is less likely to form during polymer drilling owing to its low vaporization point.

\section{MATHEMATICAL MODEL}

When a laser beam is incident on polymer substrates, a portion of the light penetrates into the material and deposits a fraction of its energy within a certain volume of the substrate. Thus the laser beam acts as a volumetric heat source. As the substrate surface temperature rises, melting and material removal due to vaporization and chemical degradation of the polymer occur creating a hole in the substrate.

\section{A. Gaussian beam propagation}

The propagation of the Gaussian laser beam in the substrate is analyzed for the drilling geometry presented in Fig. 1 showing a multilayered substrate consisting of an embedded copper layer covered with polymer layers on both sides of the copper pad. The incident laser beam is focused towards the substrate with a lens of focal length $f_{a}$ creating a beam waist $w_{0 a}$ at the focal spot. The distance between the focal spot and the top surface of the substrate is $z_{w a}$. As drilling progresses, the concave drilling front, which is simplified as a negative focusing lens, defocuses the laser beam producing another beam waist $w_{0}$ which is imaginary at the focal spot of the negative lens. If the effective focal length of

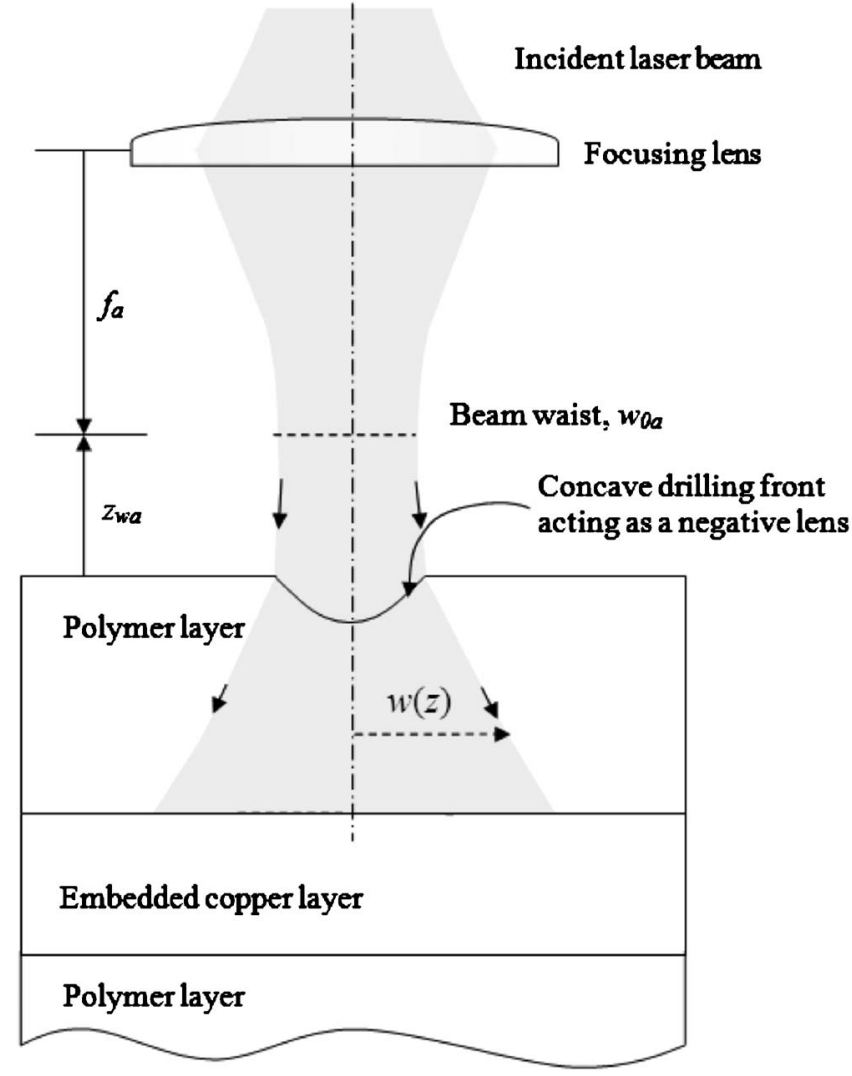

FIG. 1. Self-defocusing effect in laser drilling of semitransparent materials.

the drilling front is taken as $f$ which is negative, the beam waist $w_{0}$ can be expressed as ${ }^{20}$

$$
\frac{1}{w_{0}^{2}}=\frac{1}{w_{0 a}^{2}}\left(1+\frac{z_{w a}}{f}\right)^{2}+\frac{1}{f^{2}}\left(\frac{\pi w_{0 a}}{\lambda_{m}}\right)^{2},
$$

where $\lambda_{m}$ is the wavelength of the laser inside the polymer material, i.e., $\lambda_{m}=\lambda / n_{m}, \lambda$ is the wavelength of the laser in vacuum and $n_{m}$ is the refractive index of the polymer.

The location of the waist, $z_{w}$, is given ${ }^{20}$

$$
z_{w}=f-\left(z_{w a}+f\right) \frac{f^{2}}{\left(z_{w a}+f\right)^{2}+\left(\pi w_{0 a}^{2} / \lambda_{m}\right)^{2}},
$$

and the radius of the beam is given by

$$
w(z)=w_{0}\left[1+\frac{\left(z-z_{w}\right)^{2}}{z_{R}^{2}}\right]^{1 / 2},
$$

as it propagates through the substrate. The penetrated laser beam is absorbed by the substrate with an absorption coefficient $\mu$. Assuming the Bouguer-Lambert law to be valid, the laser irradiance propagating downward inside the polymer can be expressed as

$$
I_{i p}=I_{i} \exp [-\mu(|\bar{l}|-s)],
$$

where $\bar{l}$ is the laser propagation path vector representing the path length along the direction of laser beam propagation, $s$ is the drilling depth measured in the $\mathrm{z}$ direction and $I_{i}$ is the irradiance of the incident laser beam. If the divergence of the beam is not large, $\bar{l}$ is in the $z$ direction. For a Gaussian beam, 


$$
I_{i}=\left(1-R_{p}\right) I_{0} \exp \left[-2 r^{2} / w^{2}(z)\right] \Phi(t),
$$

where $R_{p}$ is the reflectance of the polymer, $I_{0}$ is the laser irradiance at the center of the beam, and $\Phi(t)$ is the laser pulse shape function which is considered to be rectangular in this study. $R_{p}$ depends on the incident angle of the laser beam and the laser polarization, as shown in Fig. 2. The Fresnel reflection coefficients $r_{\|}$and $r_{\perp}$ for parallel- and perpendicular-polarized lights, respectively, are given by

$$
\begin{aligned}
& r_{\|}=\frac{n_{p} \cos \theta_{i}-n_{a} \cos \theta_{t}}{n_{p} \cos \theta_{i}+n_{a} \cos \theta_{t}} \quad \text { and } \\
& r_{\perp}=\frac{n_{a} \cos \theta_{i}-n_{p} \cos \theta_{t}}{n_{a} \cos \theta_{i}+n_{p} \cos \theta_{t}},
\end{aligned}
$$

where $\theta_{i}$ and $\theta_{t}$ are the incident and refraction angles, respectively, and $n_{a}$ and $n_{p}$ are refractive indices of the air and the polymer, respectively.

The reflectance $R_{\|}$and $R_{\perp}$ for parallel- and perpendicular-polarized lights, respectively, are then given by $R_{\|}=\left|r_{\|}\right|^{2}$ and $R_{\perp}=\left|r_{\perp}\right|^{2} . R_{p}$ is taken as the average value of $R_{\|}$and $R_{\perp}$.

The laser irradiance at the center of the beam is given by

$$
I_{0}=2 P t_{p} / \pi w^{2}(z) t_{\text {on }},
$$

for a pulsed laser, where $P$ is the average laser power and $t_{p}$ and $t_{\mathrm{on}}$ are the pulse duration and pulse-on time, respectively. Combining Eqs. (4), (5), and (7), $I_{i p}$ can be expressed as

$$
I_{i p}=\frac{2 P\left(1-R_{p}\right) t_{p}}{\pi w^{2}(z) t_{o n}} \exp \left(-\frac{2 r^{2}}{w^{2}(z)}\right) \exp [-\mu(z-s)] .
$$

Due to high reflectivity of the embedded copper layer in the multilayer polymer substrate (Fig. 1), a very small amount of the laser energy is absorbed at the copper surface and the rest is reflected back into the polymer layer. The radius of the reflected beam can be expressed as

$$
w_{r}(z)=w_{0}\left[1+\frac{\left(d-z_{w}+d-z\right)^{2}}{z_{R}^{2}}\right]^{1 / 2},
$$

where $d$ is the thickness of the polymer layer. The irradiance of the reflected beam at its center $\left(I_{0 r}\right)$ is given by

$$
I_{0 r}=2\left(1-R_{p}\right) R_{\mathrm{Cu}} P t_{p} / \pi w_{r}^{2}(z) t_{\mathrm{on}},
$$

where $R_{\mathrm{Cu}}$ is the reflectance of copper. If the reflected beam is still Gaussian, the corresponding irradiance $\left(I_{r p}\right)$ is given by

$$
I_{r p}=I_{0 r} \exp \left(-\frac{2 r^{2}}{w_{r}^{2}(z)}\right) \exp [-\mu(d-s+d-z)] .
$$

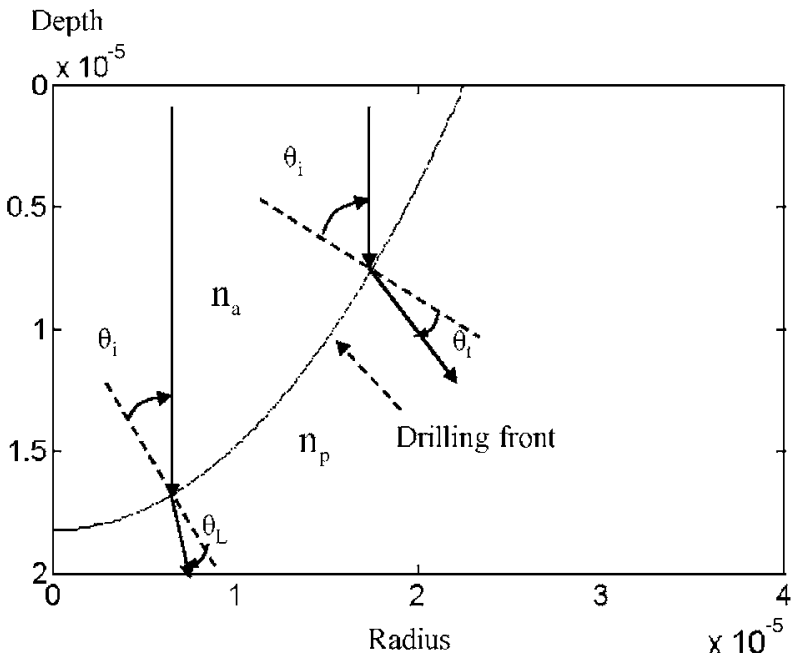

Reflectivity

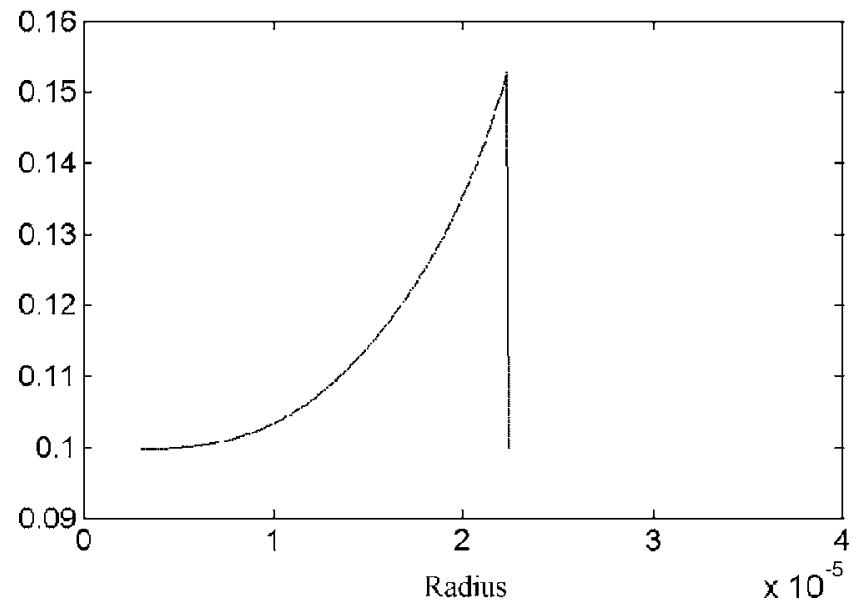

FIG. 2. Variation of reflectivity at the drilling front with the incident angle.

\section{B. Thermal model}

The thermal model for laser heating and heat conduction within the substrate is developed by assuming homogeneous and isotropic polymer material and constant thermophysical properties. The absorbed laser energy is assumed to convert into heat instantaneously. The attenuation of the beam by plasma $^{22}$ and the radiative heat loss are not considered in this model. The material removal is modeled as an ablative sublimation process, solid $\rightarrow$ vapor phase transition with chemical decomposition, because the liquid phase exists for a short duration. $^{22}$

The enthalpy method is used to solve the phase change problem, where the enthalpy in different regions of the substrate is utilized to ascertain the temperature field as given below,

$$
T= \begin{cases}T_{0}+H / C_{p}, & \text { for } H<C_{p}\left(T_{d}-T_{0}\right) \\ T_{d}, & \text { for } C_{p}\left(T_{d}-T_{0}\right) \leqslant H \leqslant C_{p}\left(T_{d}-T_{0}\right)+E_{v} \\ T_{d}+\left[H-C_{p}\left(T_{d}-T_{0}\right)+E_{v}\right] / C_{p}, & \text { for } H>C_{p}\left(T_{d}-T_{0}\right)+E_{v},\end{cases}
$$


where $T_{d}$ is the thermal decomposition temperature at which ablative material removal occurs and $E_{v}$ is the specific ablation heat. $T_{0}$ is the ambient temperature.

The transient energy equation in the cylindrical coordinate system can be written as

$$
\rho \frac{\partial H}{\partial t}=k \frac{1}{r} \frac{\partial}{\partial r}\left(r \frac{\partial T}{\partial r}\right)+k \frac{\partial^{2} T}{\partial z^{2}}+g,
$$

for $0<z<d$. Here, $k$ and $\alpha$ are the thermal conductivity and thermal diffusivity of polymer, respectively, and $g$ is the volumetric heat source given by

$$
g=-\frac{\partial I_{i p}}{\partial z}+\frac{\partial I_{r p}}{\partial z}
$$

The boundary conditions are

$$
\begin{aligned}
& k \frac{\partial T}{\partial z}=0, \quad \text { at } z=0, \\
& -k \frac{\partial T}{\partial z}+I_{a}=\frac{T-T_{0}}{R_{\text {th }}}, \quad \text { at } z=d, \\
& k \frac{\partial T}{\partial r}=0, \quad \text { at } r=0, \\
& T=T_{0}, \quad \text { at } r=\infty .
\end{aligned}
$$

$I_{a}$ is the laser irradiance absorbed by the copper surface, which is given by

$$
I_{a}=\left(1-R_{\mathrm{Cu}}\right) I_{i} \exp \{-\mu[d-s(r, t)]\} .
$$

$R_{\mathrm{th}}$ is the thermal resistance of the composite medium made of the copper layer and the polymer layer beneath the copper layer. It should be noted that the polymer layer above the copper layer is being considered for microvia drilling. The value of the thermal resistance can be obtained from the following relation:

$$
R_{\mathrm{th}}=\frac{d_{\mathrm{Cu}}}{k_{\mathrm{Cu}}}+\frac{d_{p}}{k},
$$

where $d_{\mathrm{Cu}}$ and $k_{\mathrm{Cu}}$ are the thickness and thermal conductivity of the copper layer. $d_{p}$ is the thickness of thermal penetration in the underlying polymer layer.

\section{Numerical solution}

The time-split MacCormack method ${ }^{21}$ is used to solve the partial differential equation (13) by taking the numerical stability factor $r^{\prime}=\alpha \Delta t /(\Delta r \Delta z)$ less than 0.5 , where $\Delta t, \Delta r$, and $\Delta z$ are, respectively, time step and spatial steps in the $r$ and $z$ directions. A computational flow chart is presented in Fig. 3 and various thermophysical properties of the polymer material are listed in Table I. Different laser parameters such as the wavelength, average power, beam size, pulse repetition rate, and pulse width are also input parameters for numerical calculations. The substrate surface is flat before the drilling process begins and, therefore, the effective focal length of the surface is taken as infinite prior to material removal. The drilling front, however, presents a concave sur-

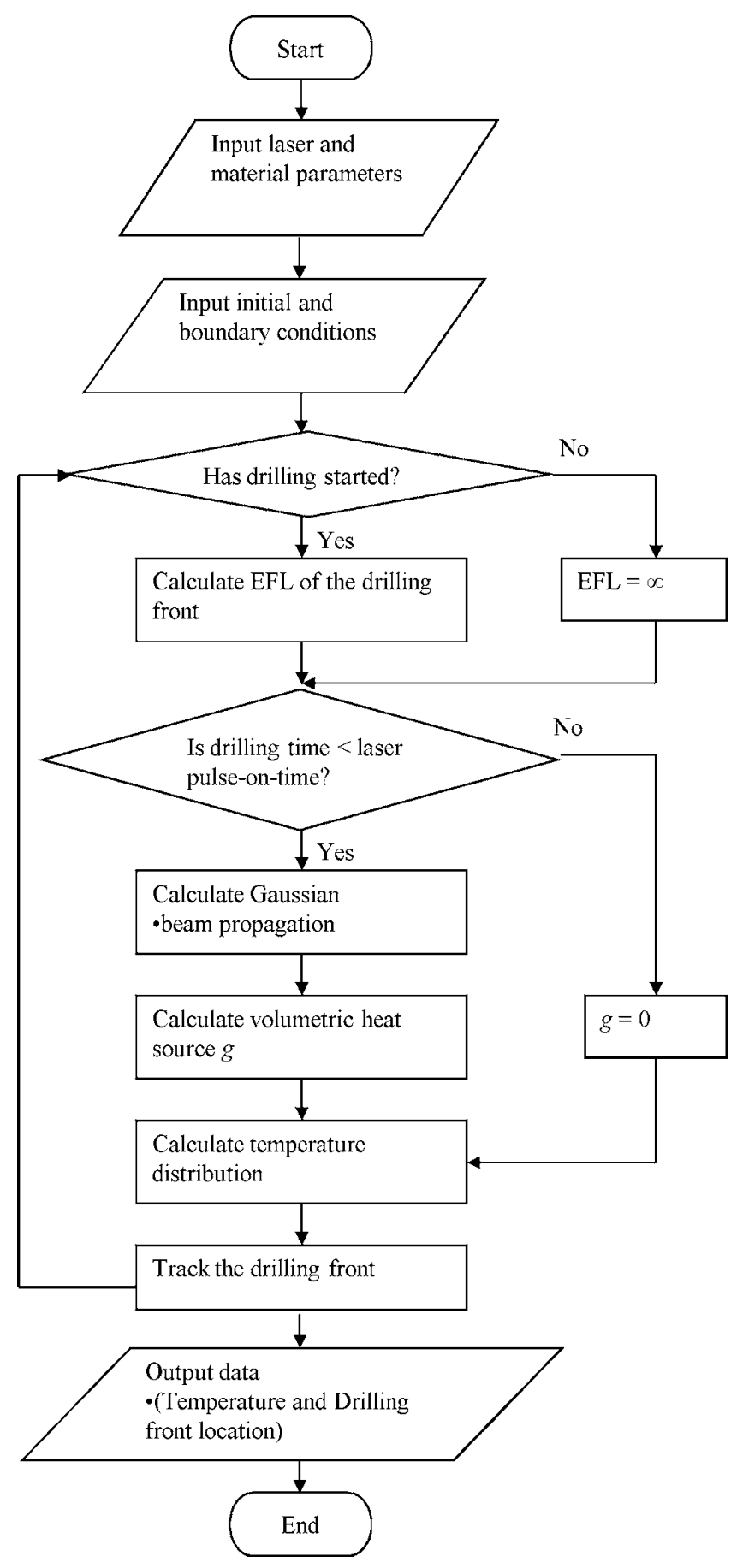

FIG. 3. Flow chart for numerical computation.

face to the incident laser beam after drilling begins to occur, for which the effective focal length is calculated by fitting the drilling front with second order polynomials such as $s(r, t)=C_{1} r^{2}+C_{2} r+C_{3}$, where $C_{1}, C_{2}$, and $C_{3}$ are constants and $f=n_{p} / 2 C_{1}\left(n_{p}-n_{a}\right)$.

The propagation of laser beam inside the material is simulated during the laser pulse-on time when the volumetric heat source $g$ is calculated based on the absorption of the laser energy given by Eq. (14). The volumetric heat source is set to zero during the laser pulse-off time. The temperature field is calculated by the time-split MacCormack method. If the temperature of a grid is larger than the thermal decomposition temperature $\left(T_{d}\right)$, that grid is considered to absorb 
TABLE I. Thermophysical and optical properties of the polymer used for numerical computations.

\begin{tabular}{lc}
\hline \hline Thermal conductivity & $0.12 \mathrm{~W} / \mathrm{m} \mathrm{K}$ \\
Specific heat & $1.62 \times 10^{3} \mathrm{~J} / \mathrm{kg} \mathrm{K}$ \\
Density & $1.44 \times 10^{3} \mathrm{~kg} / \mathrm{m}^{3}$ \\
Thermal decomposition point & $539 \mathrm{~K}$ \\
Specific ablation heat & $4.67 \mathrm{MJ} / \mathrm{kg}$ \\
Thermal expansion coefficient & $11.2 \times 10^{-5} \mathrm{~K}^{-1}$ \\
Elastic modulus & $0.62 \mathrm{GPa}$ \\
Poisson ratio & 0.35 \\
Refractive index $(\lambda=9.3 \mu \mathrm{m})$ & 1.92 \\
Refractive index $(\lambda=1.06 \mu \mathrm{m})$ & 10.63 \\
Reflectance $(\lambda=9.3 \mu \mathrm{m})$ & $9.97 \%$ \\
Reflectance $(\lambda=1.06 \mu \mathrm{m})$ & $68.56 \%$ \\
Absorption coefficient $(\lambda=9.3 \mu \mathrm{m})$ & $0.103 \mu \mathrm{m}^{-1}$ \\
Absorption coefficient $(\lambda=1.06 \mu \mathrm{m})$ & $0.041 \mu \mathrm{m}^{-1}$
\end{tabular}

the specific ablation heat according to Eq. (12). The grid is eliminated from the computational domain if the enthalpy of the grid is greater than or equal to $C_{p}\left(T_{d}-T_{0}\right)+E_{v}$, which corresponds to material removal during the drilling process. Usually, the enthalpy of a grid does not come out to be exactly equal to $C_{p}\left(T_{d}-T_{0}\right)+E_{v}$ and, therefore, the material removal (the drilling) lies somewhere between two grid points. The enthalpy is considered to vary linearly between two consecutive grid points, as shown in Fig. 4, in order to track the drilling front.

\section{RESULTS AND DISCUSSION}

The computational domain of the polymer material is $60 \mu \mathrm{m}$ in the $r$ direction and $40 \mu \mathrm{m}$ in the $z$ direction. The independence of the solution on the number of grid is tested by comparing the numerical results to an analytical model, ${ }^{23}$ as presented in Fig. 5, showing the axial temperature distribution in the polymer material at the center of the Gaussian beam $(r=0)$. Various laser parameters for this testing are the following: $3 \mathrm{~W}$ average power of a $\mathrm{CO}_{2}$ laser of wavelength of $9.3 \mu \mathrm{m}, 20 \mu$ s laser pulse-on time with a period (pulse-on time + pulse-off time) of $50 \mu \mathrm{s}$. The calculation error, which is represented by the difference between the numerical result and the analytic solution is less than $0.7 \%$ when the calculation domain is divided into $200 \times 200$ grids and the error is less than $0.2 \%$ for $500 \times 500$ grids. The results presented in this paper are for the calculation domain divided into 600 $\times 400$ grids with calculation error less than $0.3 \%$. The stability of the calculation is shown in Fig. 6, indicating that the

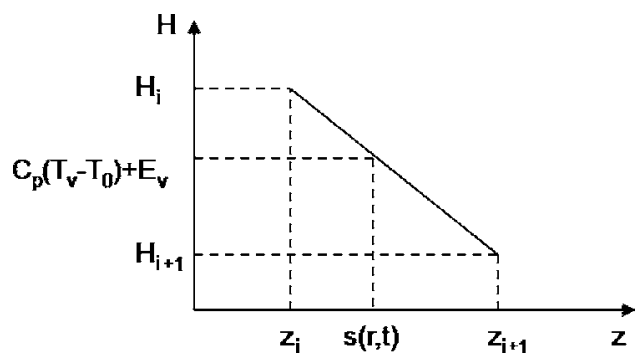

FIG. 4. Tracking the drilling front within a grid bounded by grid points $Z_{i}$ and $Z_{i+1}$.

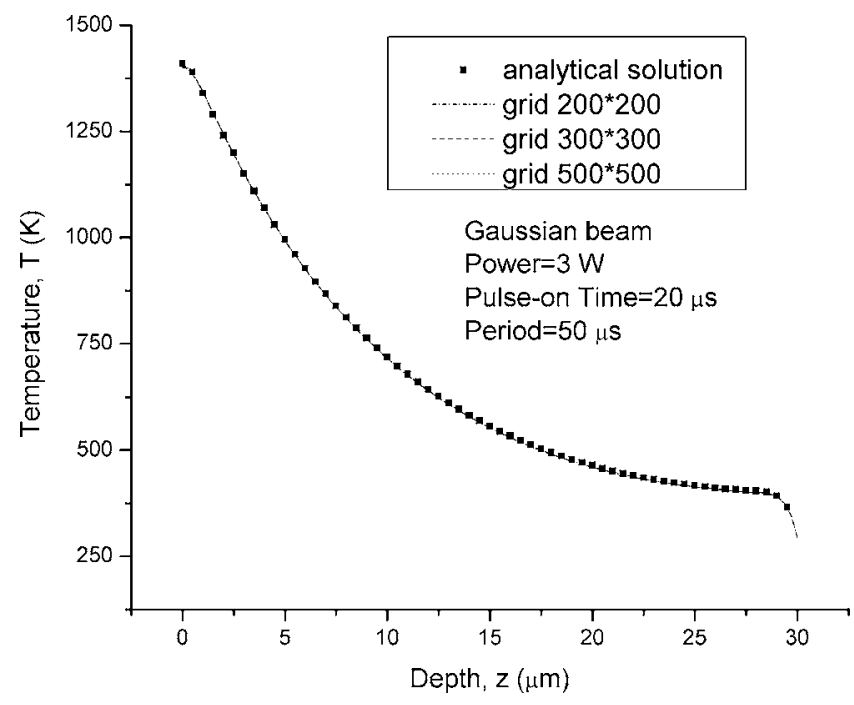

FIG. 5. Comparison of an analytic solution with numerical results for different grid numbers, indicating the selection of grids for computational accuracy.

time-split MacCormack method yields a stable solution even when the stability factor $r^{\prime}=\alpha \Delta t /(\Delta r \Delta z)$ is as large as 0.47 .

The temperature distributions along the $z$ direction at $r$ $=0$ are presented in Fig. 7 for four different times after the beginning of laser irradiation. Because of the Gaussian beam profile, the maximum laser irradiance $\left(I_{0}\right)$ occurs at the center of the beam $(r=0)$ and this produces maximum temperature at $r=0$ on a given plane of fixed depth. The energy of the Gaussian laser beam is $0.392 \mathrm{~mJ} /$ pulse, beam radius is $25 \mu \mathrm{m}$, pulse-on time is $430 \mathrm{~ns}$, and the pulse repetition rate is $50 \mathrm{kHz}$. As the irradiation time increases, the temperature inside the polymer increases. After the thermal decomposition point is reached, the temperature will not rise because the specific ablation heat is absorbed. Since the polymer material can greatly attenuate the laser beam propagating inside it, the temperature is maximum at the top surface and drilling begins from top to the bottom for $\mathrm{CO}_{2}$ lasers. In this paper, the enthalpy method is used to describe the phase change and

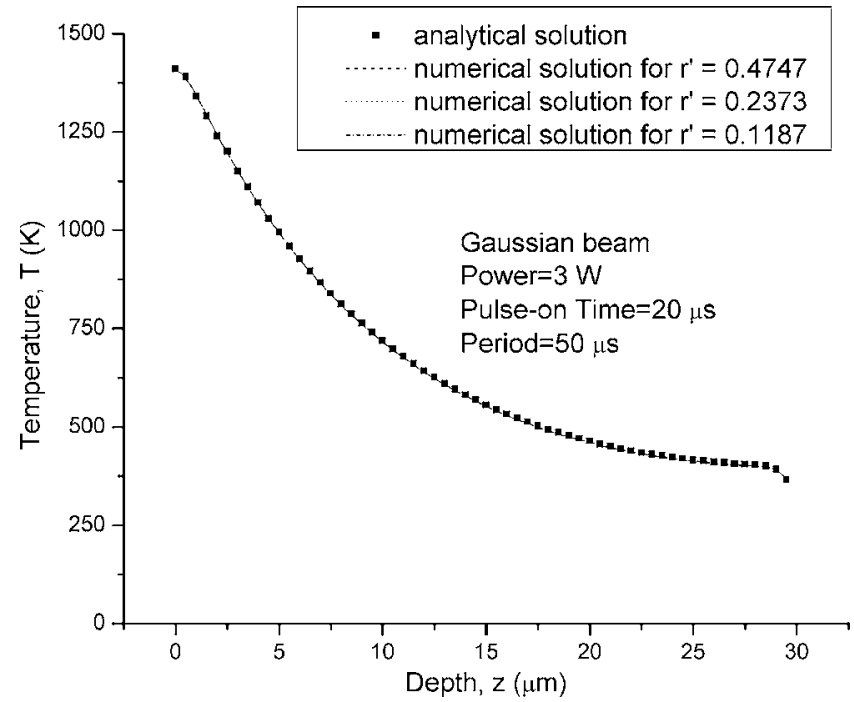

FIG. 6. Comparison of numerical results for different time step, indicating the selection of the time step for computational stability. 


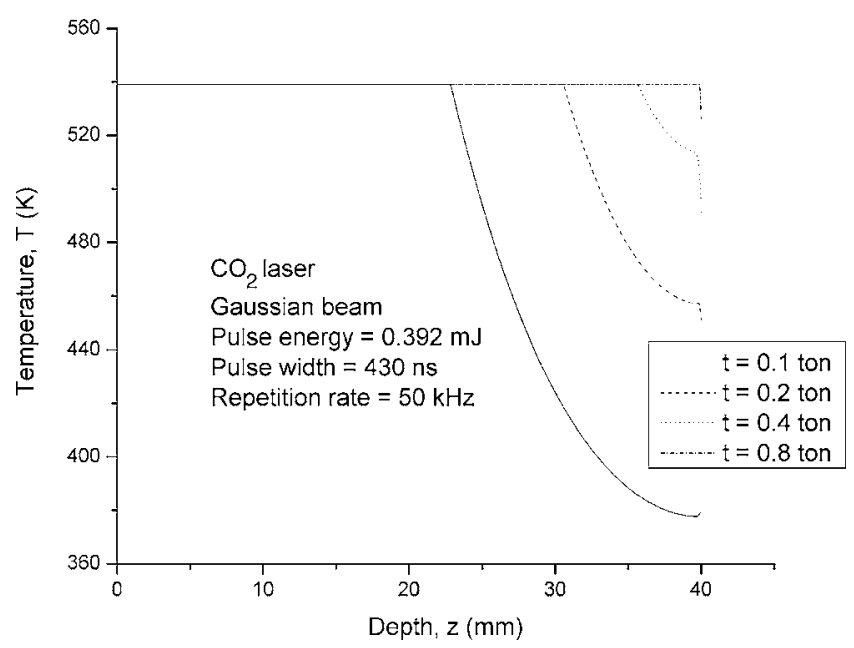

FIG. 7. Axial temperature distribution at different times during $\mathrm{CO}_{2}$ laser drilling.

the laser heating model is not based on the boundary condition of a fixed temperature at the drilling front. Consequently, overheating of the polymer, i.e., the occurrence of maximum temperature inside the material, is not seen in this case (Fig. 7). However, if the temperature is held constant at the driiling front, overheating can be observed inside the polymer substrate. $^{2}$

However, the drilling mechanism is different for Nd:YAG lasers of wavelength of $1.06 \mu \mathrm{m}$. The axial temperature distribution due to Nd:YAG laser irradiation is compared to that of the $\mathrm{CO}_{2}$ laser in Fig. 8 at time $t=0.1 t_{\text {on }}$ which is $43 \mathrm{~ns}$. The laser parameters are the same as those used in the case of the $\mathrm{CO}_{2}$ laser irradiation. Since the absorption coefficient of the polymer material is $0.041 \mu \mathrm{m}^{-1}$ at the wavelength of the Nd:YAG laser, which is less than that $\left(0.103 \mu \mathrm{m}^{-1}\right)$ at the $\mathrm{CO}_{2}$ laser wavelength as listed in Table I, the Nd:YAG laser energy reaching the polymer-copper interface will be higher than in the case of the $\mathrm{CO}_{2}$ laser irradiation. This causes the temperature at the copper-polymer interface to be higher for the Nd:YAG laser than for the $\mathrm{CO}_{2}$

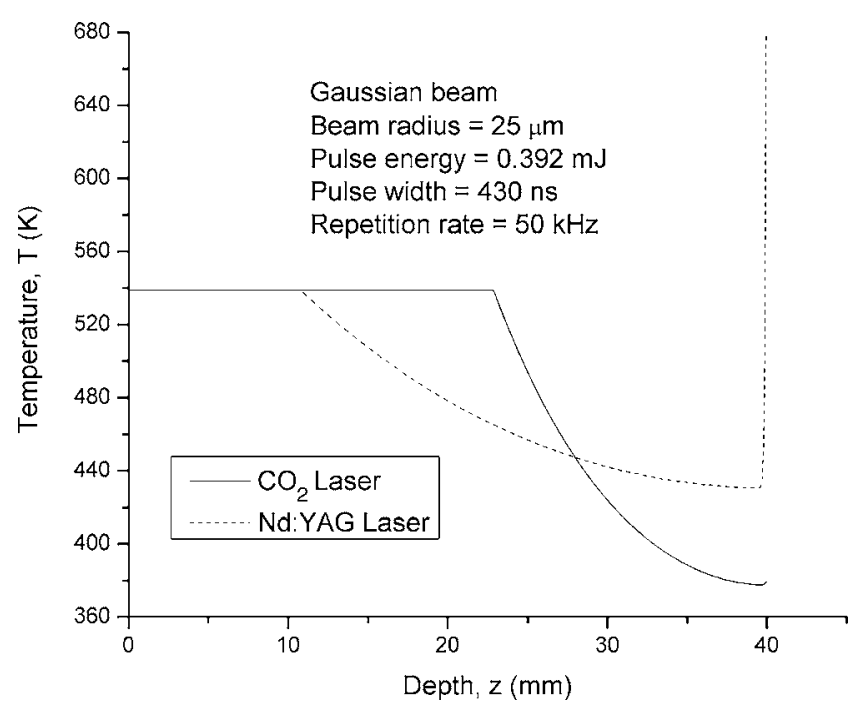

FIG. 8. Comparison of the axial temperature distributions for Nd:YAG and $\mathrm{CO}_{2}$ laser drillings at $r=0$ and drilling time $t=0.1 t_{\text {on }}$.

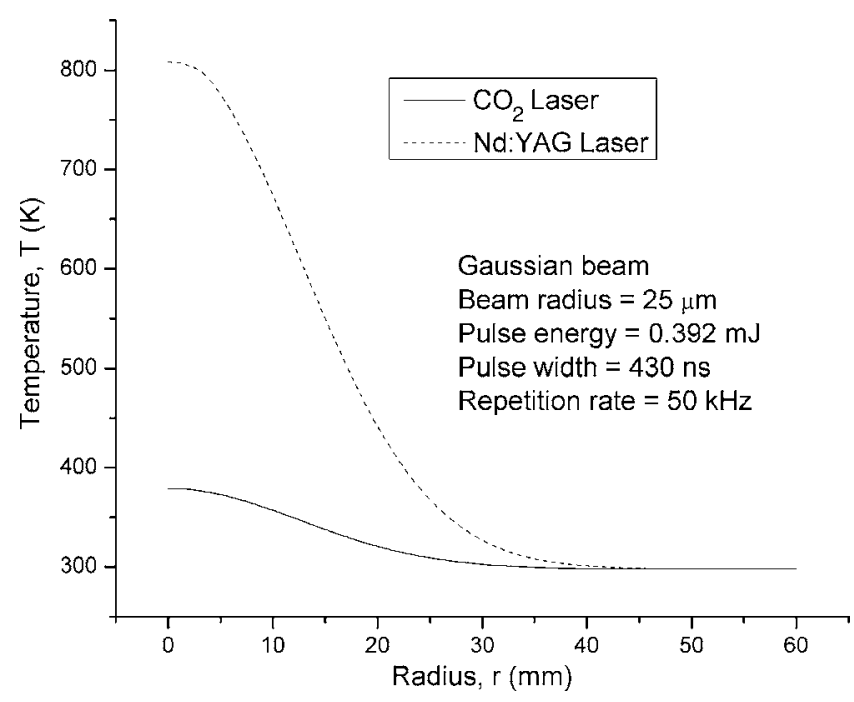

FIG. 9. Comparison of the radial temperature distributions for Nd:YAG and $\mathrm{CO}_{2}$ laser drillings at the interface of the top polymer and copper layers when drilling time $t=0.1 t_{\mathrm{on}}$.

laser. The radial temperature distribution at the interface is presented in Fig. 9 at time $t=0.1 t_{\mathrm{on}}$, which shows that the temperature will be higher than the thermal decomposition point within a radius of $15 \mu \mathrm{m}$ after $43 \mathrm{~ns}$ of laser irradiation. The polymer may turn into vapor in this region, resulting in high pressure and high thermal stresses inside the material. If the pressure and thermal stresses are high enough, the polymer material above the copper layer will be expelled out and a hole will be formed. This type of material expulsion can improve the drilling rate because more material can be removed by the expulsion process than just by vaporizing the material only at the drilling front.

The drilling front profiles formed at different times are presented in Fig. 10 for Gaussian $\mathrm{CO}_{2}$ lasers with pulse energy of $0.392 \mathrm{~mJ}$, beam radius of $25 \mu \mathrm{m}$, pulse-on time of $430 \mathrm{~ns}$, and repetition rate of $50 \mathrm{kHz}$. The hole depth increases as the drilling time increases and the embedded copper layer can be reached after three pulses. More pulses can

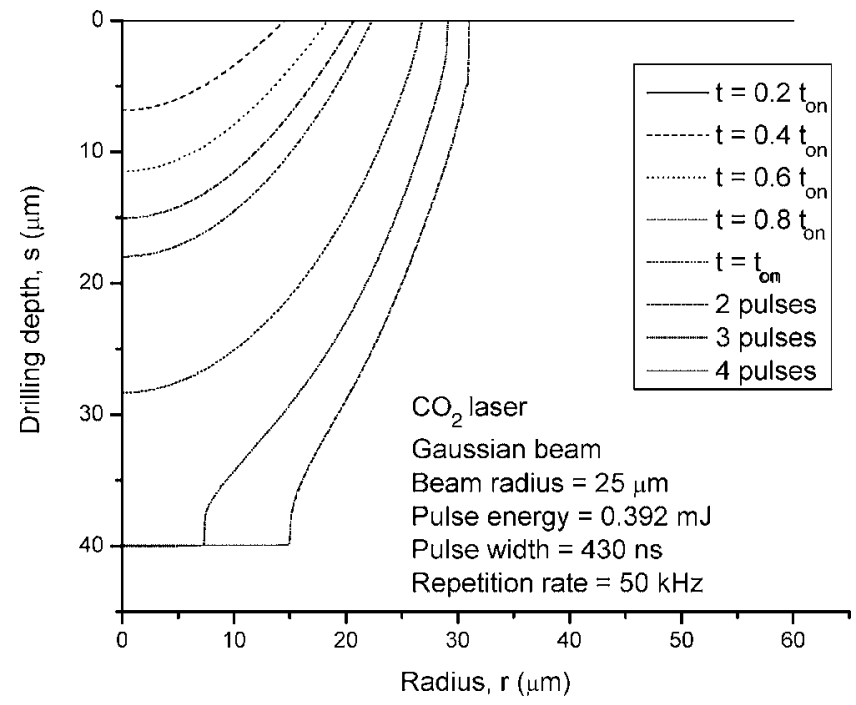

FIG. 10. Depth of the drilling front at different radial locations (drilling front profile) as the drilling progresses. 


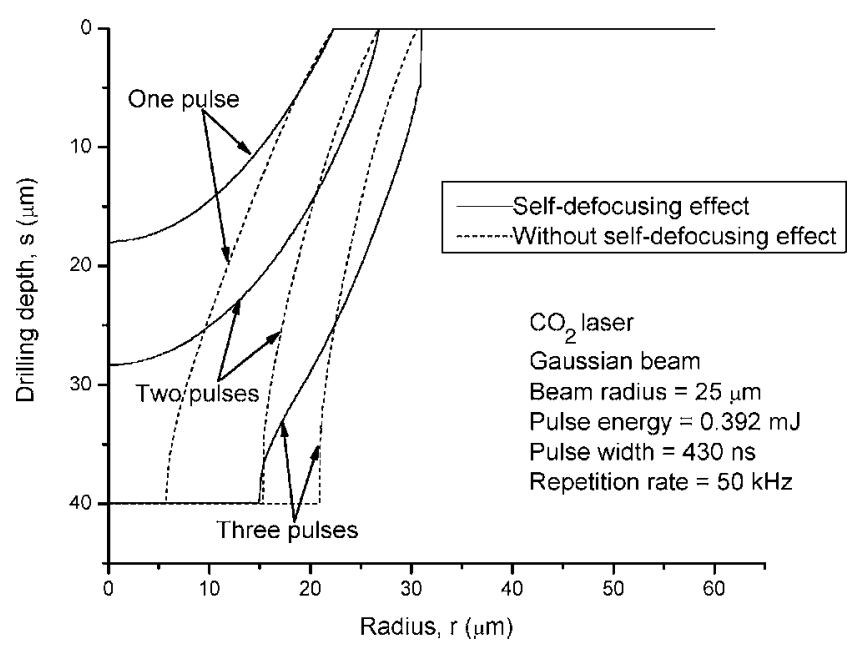

FIG. 11. Self-defocusing effect on the drilling front profile.

increase the hole diameter at the copper surface and thus reduce the tapering angle. The self-defocusing effect discussed in this paper can affect the drilling process significantly as seen from the results in Fig. 11. Without the selfdefocusing effect, the embedded copper layer can be reached after one laser pulse which is less than three laser pulses required with the self-defocusing effect.

The drilling is not initiated instantaneously after the laser irradiation begins. The lower the pulse energy, the longer time is needed to initiate the drilling, as shown in Fig. 12. During this time, the laser energy heats up the material to the thermal decomposition point and supply the specific ablation heat. This energy is termed as the ablation threshold energy. The ablation threshold fluence is calculated for different pulse energies in Fig. 13. Although the drilling inception time is different in Fig. 12, the laser energy (i.e., the product of the drilling time and the average laser power) supplied to the substrate are the same and the corresponding ablation threshold energies are the same for different pulse energies, as shown in Fig. 13. When the laser energy is accumulated in

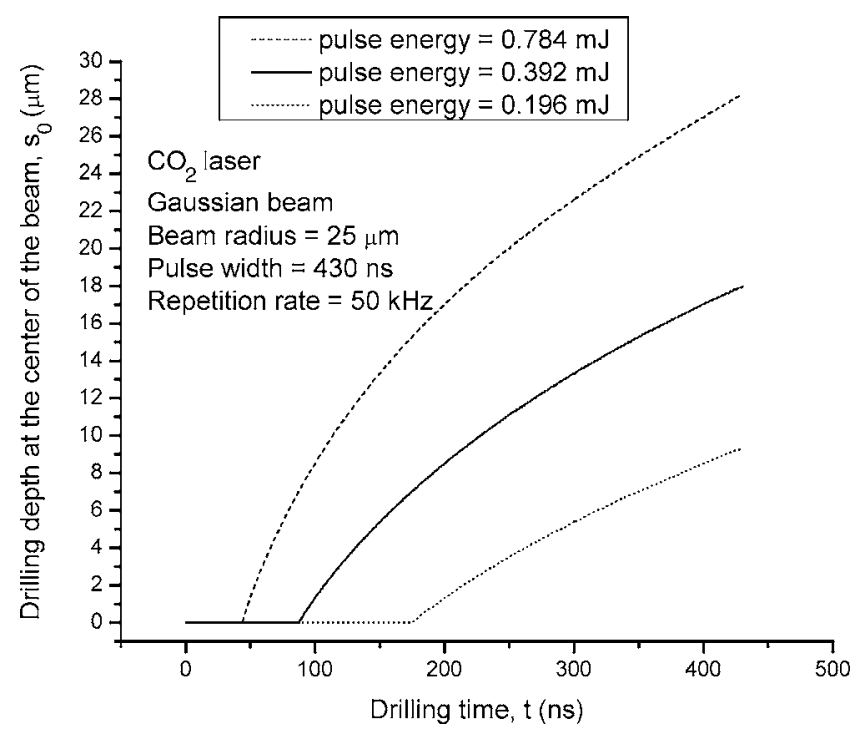

FIG. 12. Drilling depth at the center of the laser beam as a function of time for different $\mathrm{CO}_{2}$ laser pulse energies.

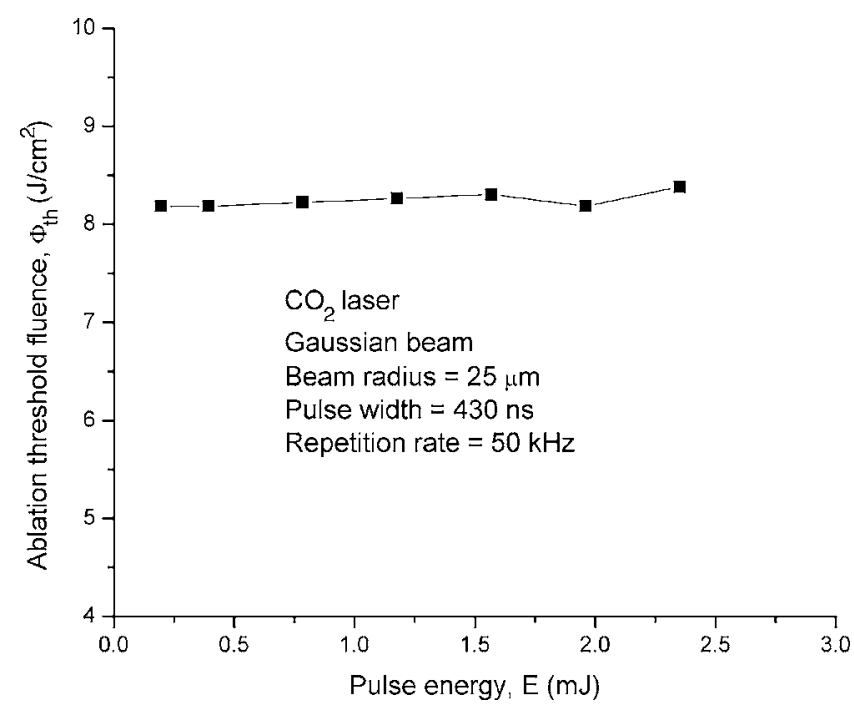

FIG. 13. Ablation threshold fluence for different $\mathrm{CO}_{2}$ laser pulse energies.

the material for being heated up to the ablation threshold energy, the absorbed energy may also be lost due to heat diffusion. The diffusion heat loss largely depends on the drilling time. Longer diffusion time means large heat loss. Since the ablation threshold energy is found to be the same in Fig. 13 for different nanosecond laser pulses, the diffusion heat loss is negligible in these cases as nanosecond is a small time period for the occurrence of heat diffusion.

The effect of laser beam shape on the drilling process is also analyzed for three types of beam shapes, uniform beam, Gaussian beam, and pitchfork beam, as shown in Fig. 14. For uniform beams, the beam shape at the focal spot is a Bessel beam ${ }^{24}$ due to diffraction. The drilling front profiles due to Bessel beam, Gaussian beam and pitchfork beam are calculated using the numerical model. The laser parameters for these three types of beam shapes are the following: pulse energy of $0.392 \mathrm{~mJ} /$ pulse, beam radius of $25 \mu \mathrm{m}$, pulse-on time of $430 \mathrm{~ns}$, and pulse repetition rate of $50 \mathrm{kHz}$. The drilling front profiles are shown in Fig. 15 for the three beam shapes after the first pulse. For the same amount of laser energy, the pitchfork beam has the possibility of producing microvias with the smallest tapering angle. The advantage of

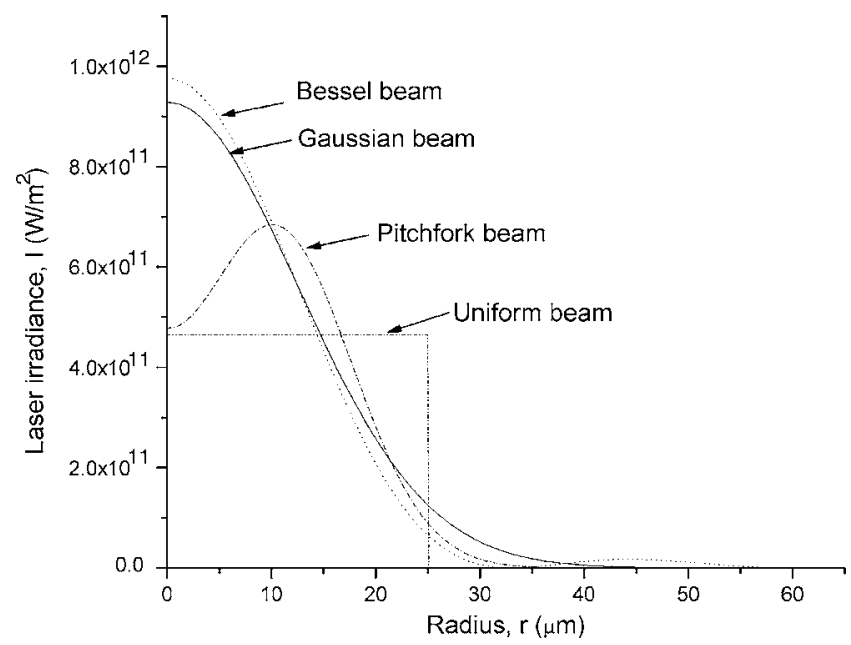

FIG. 14. Different laser irradiances used in the comparison of drilling front profiles. 


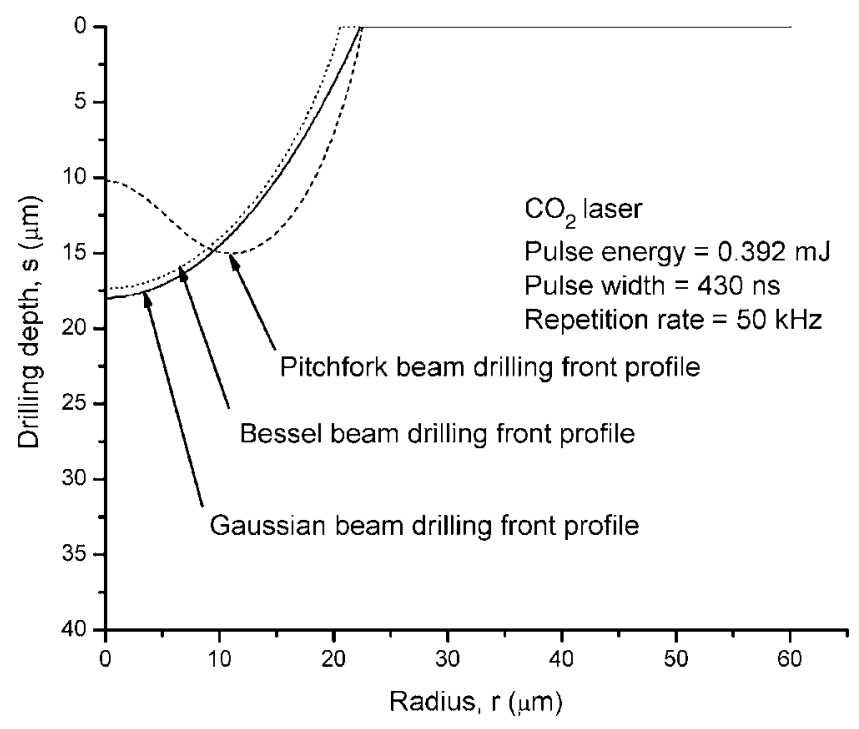

FIG. 15. Comparison of drilling front profiles after one laser pulse for different beam shapes with the same pulse energy.

the pitchfork beam is that it can utilize the self-defocusing or self-focusing effect to improve the effectiveness of the drilling process. The central region of the drilling front in Fig. 15 can be regarded as a positive focusing lens for the pitchfork beam, whereas the same region will act as a negative lens for the Gaussian beam. Therefore, the central portion of the pitch fork beam will be focused by the drilling front, raising its irradiance and causing rapid material removal. Thus, the drilling speed is enhanced by the pitchfork beam. Drilling experiment was carried out with the pitchfork laser beam. A scanning electron microscopic cross-sectional view of the microvia drilled by a pitchfork laser beam is shown in Fig. 16. The laser parameters for the drilling experiment were as

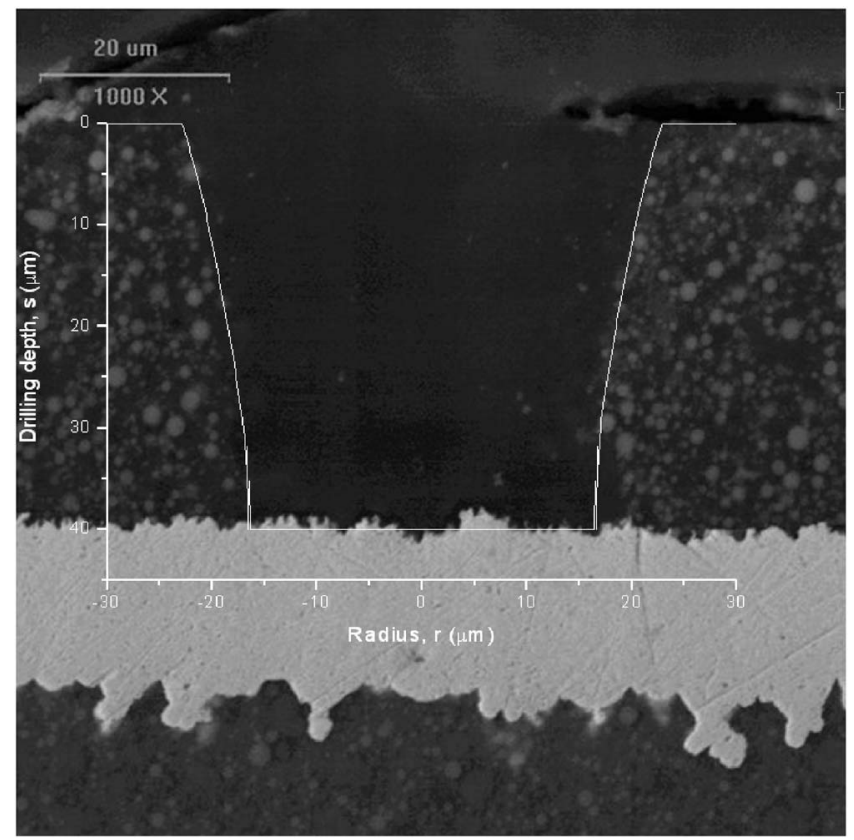

FIG. 16. Drilling front profile by pitchfork laser beam (pulse energy $=0.3 \mathrm{~mJ}$, beam radius $=21 \mu \mathrm{m}$, pulse width $=430 \mathrm{~ns}$, repetition rate $=83.3 \mathrm{kHz}$ ). Due to diffraction the central lobe of the beam only contains $18.9 \%$ of the total laser energy. follows: pulse energy $=0.3 \mathrm{~mJ}$, beam radius $=21 \mu \mathrm{m}$, pulse width $=430 \mathrm{~ns}$, and repetition rate $=83.3 \mathrm{kHz}$. The optics to convert an incident Gaussian beam into the pitchfork beam was so designed that the central valley and peripheral peak regions (Fig. 14) of the beam contained $1.1 \%$ and $17.8 \%$ of the total laser energy, respectively. Eight pulse trains each containing eight pulses were used to drill one microvia. The drilling front profile calculated by the model with the same laser parameters is also shown in Fig. 16 as the white solid line which agrees the experimental data very well.

\section{CONCLUSION}

A numerical thermal model is developed accounting for the self-defocusing effect to analyze the microvia drilling process in polymer substrates.

(1) Self-defocusing of the laser beam by the drilling front can greatly reduce the drilling speed. So the refractive index of the material at a specific wavelength is an important parameter for laser drilling.

(2) The $\mathrm{CO}_{2}$ laser drilling of polymer substrates is mainly due to ablation as well as vaporization. The Nd:YAG laser drilling mechanism involves thermomechanical breakage or expulsion of the material by high internal pressure.

(3) The self-focusing effect can be utilized advantageously with pitch fork beams to increase the drilling speed compared to uniform and Gaussian beam shapes.

\section{ACKNOWLEDGMENT}

This work was supported by AppliCote Associates, LLC.

${ }^{1}$ L. G. Hector and R. B. Hetnarski, Thermal Stresses IV (Elsevier, New York, 1996), p. 453.

${ }^{2}$ C. Zhang, I. A. Salama, N. R. Quick, and A. Kar, J. Appl. Phys. 99, 113530 (2006).

${ }^{3}$ M. Olfert and W. W. Duley, J. Phys. D 29, 1140 (1996).

${ }^{4}$ R. Halter, S. Jayaraman, and B. R. Tittmann, Proc. SPIE 4336, 197 (2001).

${ }^{5}$ A. Kar, T. Rockstroh, and J. Mazumder, J. Appl. Phys. 71, 2560 (1992).

${ }^{6}$ A. Kar and J. Mazumder, Phys. Rev. E 49, 410 (1994).

${ }^{7}$ A. Kar, J. E. Scott, and W. P. Latham, J. Appl. Phys. 80, 667 (1996).

${ }^{8}$ J. Xie and A. Kar, J. Appl. Phys. 81, 3015 (1997).

${ }^{9}$ W. Pecharapa and A. Kar, J. Phys. D 30, 3322 (1997).

${ }^{10}$ H. Ki, P. S. Mohanty, and J. Mazumder, J. Laser Appl. 14, 39 (2002).

${ }^{11}$ A. Ruf, P. Berger, F. Dausinger, and H. Hugel, J. Phys. D 34, 2918 (2001).

${ }^{12}$ S. Noguchi, E. Ohmura, and I. Miyamoto, Proc. SPIE 4830, 46 (2003).

${ }^{13}$ K. T. Voisey and T. W. Clyne, Surf. Coat. Technol. 176, 296 (2004).

${ }^{14}$ H. K. Sezer, L. Li, M. Schmidt, A. J. Pinkerton, B. Anderson, and P. Williams, Int. J. Mach. Tools Manuf. 46, 1972 (2006).

${ }^{15}$ V. V. Semak, B. Damkroger, and S. Kempka, J. Phys. D 32, 1819 (1999).

${ }^{16}$ D. Zeng, W. P. Latham, and A. Kar, J. Appl. Phys. 97, 104912 (2005).

${ }^{17}$ D. Ashkenasi, H. Varel, A. Rosenfeld, S. Henz, J. Herrmann, and E. E. B. Cambell, Appl. Phys. Lett. 72, 1442 (1998).

${ }^{18}$ J. Sun and J. P. Longtin, J. Opt. Soc. Am. B 21, 1081 (2004).

${ }^{19}$ Amrita and A. K. Sharma, Phys. Scr. 74, 128 (2006).

${ }^{20}$ P. Strombeck and A. Kar, J. Phys. D 31, 1438 (1998).

${ }^{21}$ D. A. Anderson, J. C. Tannehill, and R. H. Pletcher, Computational Fluid Mechanics and Heat Transfer (Hemisphere, New York, 1984), p. 166.

${ }^{22}$ C. Zhang, S. Bet, I. A. Salama, N. R. Quick, and A. Kar, Symp. Packaging of Electronic and Photonic Systems, InterPack, 2005 (American Society of Mechanical Engineers, New York, 2005).

${ }^{23}$ C. Zhang, I. A. Salama, N. R. Quick, and A. Kar, Symposium of ICALEO 2005 (Laser Institute of America, Orlando, FL, 2005), p. 404.

${ }^{24}$ E. Hecht, Optics (Addison Wesley, New York, 2001), p. 480. 